POS $\quad$ PROCEEDINGS

\title{
Astronomical X-ray Telescopes and Monitors of Lobster Eye Type
}

\author{
Rene Hudec*t \\ Astronomical Institute of the Academy of Sciences of the Czech Republic \\ CZ-251 65 Ondrejov, Czech Republic, and \\ Czech Technical University in Prague, Faculty of Electrical Engineering \\ Prague, Czech Republic E-mail: rhudec@asu.cas.cz
}

\section{Ladislav Pina}

Czech Technical University, Prague

E-mail: pina@fjfi.troja.cuni.cz

\section{Libor Švéda}

Czech Technical University, Prague

E-mail: vojtech.simon@gmail.com

\section{Adolf Inneman}

Rigaku Innovative Technologies Europe, Prague

E-mail: adolf.inneman@rigaku.com

\section{Veronika Maršiová}

Rigaku Innovative Technologies Europe, Prague

E-mail: veronika.marsikova@rigaku.com

\section{Vojtěch Šimon}

Astronomical Institute of the Academy of Sciences of the Czech Republic CZ-251 65 Ondrejov, Czech Republic

E-mail: simoneasu.cas.cz

We report on technical as well as astrophysical aspects of very wide-field X-ray telescopes with high sensitivity. The prototypes constructed so far are promising, allowing the proposals for space projects with very wide-field Lobster-Eye (LE) X-ray optics to be considered. The LE telescopes will monitor the sky with unprecedented sensitivity and angular resolution of order of 1 arcmin. They are expected to contribute essentially to study of various astrophysical objects such as AGN, SNe, Gamma-ray bursts (GRBs), X-ray flashes (XRFs), galactic binary sources including microquasars, stars, CVs, X-ray novae, various transient sources, etc. For example, the Lobster optics based X-ray All Sky Monitor is capable to detect around 20 GRBs and 8 XRFs yearly and this will surely significantly contribute to the related science.

The Extreme sky: Sampling the Universe above $10 \mathrm{keV}$ - extremesky2009,

October 13-17, 2009

Otranto (Lecce) Italy

* Speaker.

${ }^{\dagger}$ A footnote may follow. 


\section{Introduction}

The X-ray sky including known and yet unknown microquasars is highly variable, rich in variable and transient sources of both galactic as well as extragalactic origin. Among physically most important transient sources, the detection of Gamma Ray Bursts (GRBs) in X-rays confirms the feasibility of monitoring, detecting and study of these phenomena by their X-ray emission (either prompt or afterglow, e.g. Amati et al., [1] and Fontera et al., [5]). For classical GRBs, the $\mathrm{X}$-ray afterglows are detected in $\sim 90 \%$ of the cases (De Pasquale et al., [3]). Moreover, there are X-ray rich GRBs, (hypothetical) orphan GRBs (detectable in X-rays but not in gamma-rays due to different beaming angle) and XRFs which can be detected and studied in X-rays. However, since these events cannot be predicted, and are relatively rare, very wide-field instruments are required. They must achieve high sensitivities and provide precise localizations in order to effectively study the objects. Wide field X-ray telescopes with imaging optics are expected to represent an important tool in future space astronomy projects in general, especially those for deep monitoring and surveys in X-rays over a wide energy range. The Lobster-Eye wide field X-ray optics has been suggested in the 1970s by Schmidt (Schmidt, [10], orthogonal stacks of reflectors) and by Angel (Angel, [2], array of square cells). Up to $180 \mathrm{deg}$ FOV may be achieved. This novel X-ray optics offers an excellent opportunity to achieve very wide fields of view (FOV, 1000 square degrees and more) while the widely used classical Wolter grazing incidence mirrors are limited to roughly 1 deg FOV (Priedhorsky et al., [9], Inneman et al., [7]).

\section{Lobster Eye X-ray Telescopes}

The Lobster-Eye (LE) telescopes are based on grazing incidence optics analogous to those in eyes of lobsters and crayfish (Figs. 1, 2). Two basic types of Lobster Eye Wide Field X-ray telescopes have been proposed for astrophysical applications. The telescopes in Schmidt arrangements are based on perpendicular arrays of double-sided X-ray reflecting flats. In the first prototypes developed and tested, double-sided reflecting flats produced by epoxy sandwich technology as well as gold coated glass foils have been used (Inneman et al., [8], see also Table 1). More recently, micro Schmidt lobster eye arrays with foils thickness as low as 30 microns have been developed and tested in order to confirm the capability of these systems to achieve angular resolutions of order of a few arcmin. The thin foils are separated by 70 microns gaps in these prototypes. On the other hand, large lobster eye systems with Schmidt geometry have been designed and constructed, achieving dimensions up to $300 \times 300 \times 600 \mathrm{~mm}$ (Fig. 3). Their optical tests have confirmed the expected performance according to calculations (computer ray-tracing). The calculations and the measurement results indicate that the lobster eye telescope based on multi array of modules with thin and closely spaced glass foils (analogous to those already assembled and tested) can meet the requirements of $\mathrm{X}$-ray survey and monitoring missions (including the angular resolution and with better transmission) and can hence represent an alternative to the recently suggested MCP technique (Fraser et al., [4]).

The Angel LE optics represent the direct analogy of lobster eye. For these lenses, numerous square cells of very small size (about $1 \times 1 \mathrm{~mm}$ or less at lengths of order of tens of mm, i.e. with the length/size ratio of 30 and more) are to be produced. This demand can be also solved by mod- 


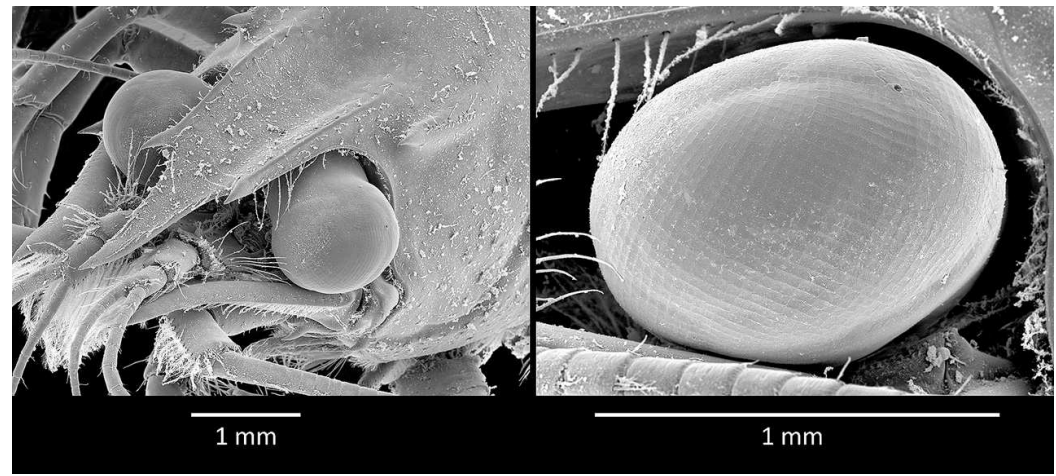

Figure 1: The crayfish head with two reflective eyes (left) and the crayfish eye (right).

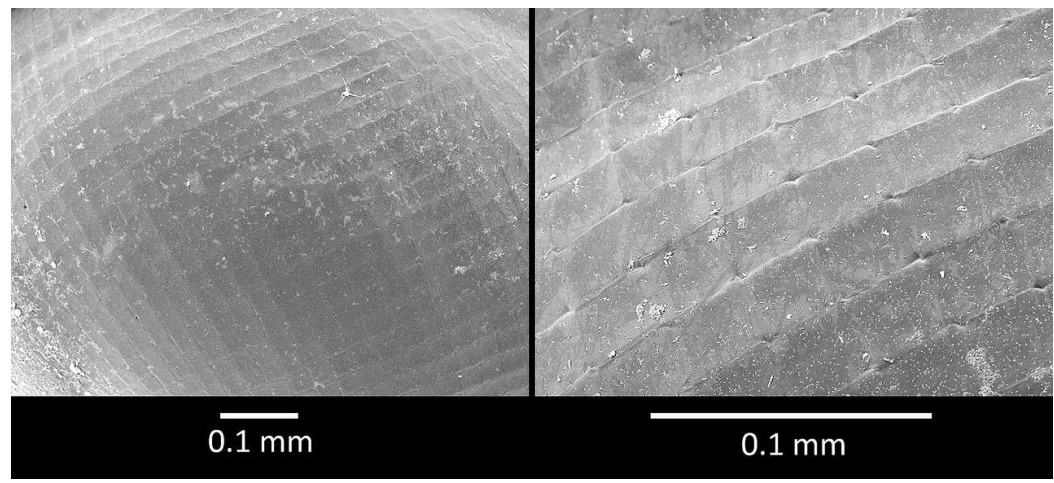

Figure 2: The details of the surface of crayfish eye showing the entrance aperture i.e. numerous square cells.

ified innovative replication technology. Test modules with LE Angel cells have been successfully produced. The linear test module has 47 cells $2.5 \times 2.5 \mathrm{~mm}, 120 \mathrm{~mm}$ long (i.e. length/size ratio of almost 50), surface microroughness $0.8 \mathrm{~nm}, f=1300 \mathrm{~mm}$. Another test module is represented by a L-shaped array of $2 \times 18=36$ cells of analogous dimension. The surface microroughness of the replicated reflecting surfaces is better than $1 \mathrm{~nm}$.

From the technological point of view, the fact that the modular concepts of Schmidt LE modules, of the large segmented Wolter telescopes (such as IXO), and of large segmented K-B (Kirkpatrick-Baez) telescopes are similar is important: all are based on either planparalel or curved flats and foils. This means that the development of high quality X-ray reflecting foils and flats with high mechanical stiffness and low volume density is extremely important for most of the future X-ray astronomy large aperture projects. The segmented $\mathrm{K}-\mathrm{B}$ telescopes have the advantage of being highly modular on several levels. All segments are rectangular boxes with the same outer dimensions (Gorenstein, [6]).

\section{Science with LE telescopes and monitors}

Deep (limiting flux of $10^{-12} \mathrm{erg} \mathrm{cm}^{-2} \mathrm{~s}^{-1}$ can be easily achieved for daily scanning observation) 
Table 1: The single Lobster Eye Schmidt modules developed so far by our collaboration. Here plates have dimensions of $d \times l \times t$ and are arranged with spacing $a$. The modules have focal length $f$ and field of view FOV and are optimized for the energy given in the last column.

\begin{tabular}{|l|c|c|c|c|c|c|c|c|}
\hline Module & $\begin{array}{c}\text { size } \\
\mathrm{d}[\mathrm{mm}]\end{array}$ & $\begin{array}{c}\text { thickness } \\
\mathrm{t}[\mathrm{mm}]\end{array}$ & $\begin{array}{c}\text { distance } \\
\mathrm{a}[\mathrm{mm}]\end{array}$ & $\begin{array}{c}\text { length } \\
1[\mathrm{~mm}]\end{array}$ & $\begin{array}{c}\text { foc. length } \\
\mathrm{f}[\mathrm{mm}]\end{array}$ & $\begin{array}{c}\text { resolution } \\
\mathrm{r}[\operatorname{arcmin}]\end{array}$ & $\begin{array}{c}\text { FOV } \\
{\left[{ }^{\circ}\right]}\end{array}$ & $\begin{array}{c}\text { energy } \\
{[\mathrm{keV}]}\end{array}$ \\
\hline macro & 300 & 0.75 & 10.80 & 300 & 6000 & 7 & 16 & 3 \\
middle & 80 & 0.3 & 2 & 80 & 400 & 20 & 12 & 2 \\
mini 1 & 24 & 0.1 & 0.3 & 30 & 900 & 2 & 5 & 5 \\
mini 2 & 24 & 0.1 & 0.3 & 30 & 250 & 6 & 5 & 5 \\
micro & 3 & 0.03 & 0.07 & 14 & 80 & 4 & 3 & 10 \\
\hline
\end{tabular}
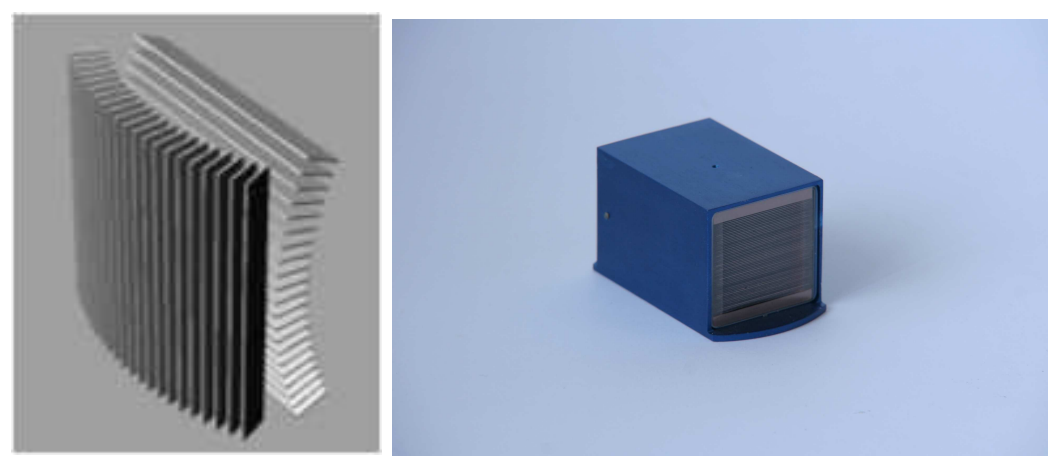

Figure 3: The principle of Lobster Eye Optics in Schmidt arrangement (left) and the LE Schmidt Mini test module (right).
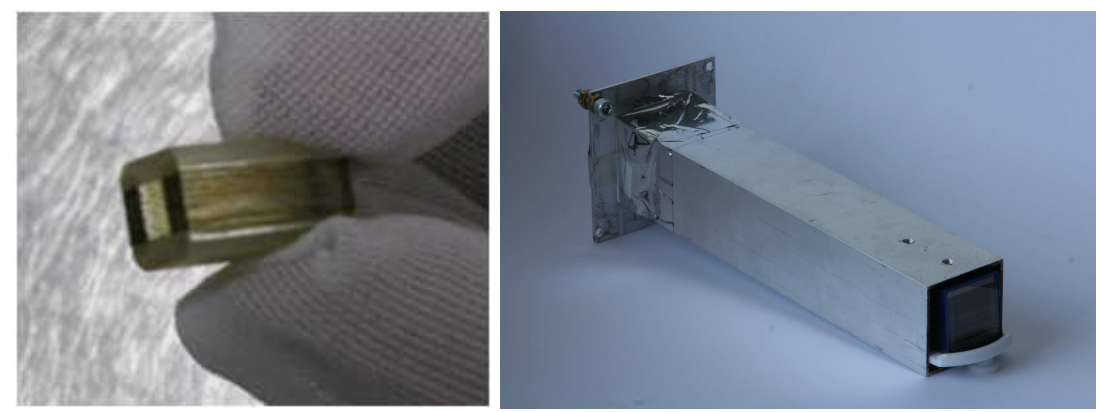

Figure 4: The Micro LE test module (left) and the LE Schmidt telescope with $\mathrm{f}=250 \mathrm{~mm}$ (right).

$\mathrm{X}$-ray sky monitoring with large FOVs (e.g. FOV of $6 \times 180 \mathrm{deg}$ can be easily assembled on the space station ISS) is expected to contribute significantly to various fields of modern astrophysics. A few most important examples are listed below.

(1) Gamma Ray Bursts (GRBs). Detection rates of nearly 20 GRBs/year can be obtained for the prompt X-ray emission of GRBs, taking into account the expected GRB rate 300/year. (2) $\mathrm{X}$-ray flashes. Detection rates of nearly $8 \mathrm{X}$-ray flashes/year are expected, assuming XRF rate of 100/year. (3) X-ray binaries. Because of their high variability in X-rays they will be one of 

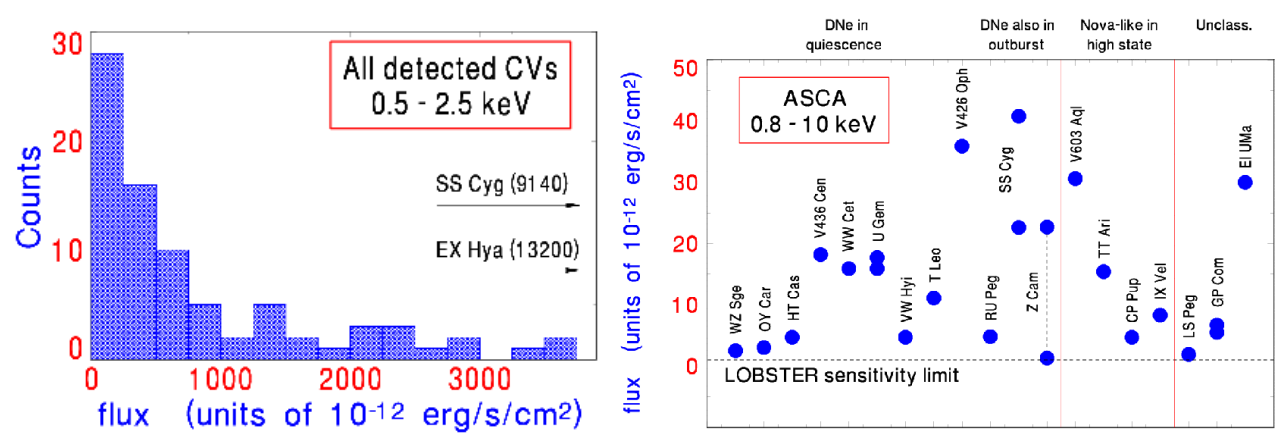

Figure 5: The numbers of all known cataclysmic variables (CVs) observable by Lobster Eye All-Sky Monitor (ASM, (left) and the various types of Dwarf Novae within the sensitivity limit of LOBSTER ASM (right).

major targets in LE observations. LE will be able to observe their short-time outbursts by longterm extended monitoring. Almost all galactic XRB are expected to be within the detection limits. (4) Stars. Because of the low X-ray luminosity of ordinary stars, only nearby stars are expected to be observable. We estimate the lower limit of ordinary stars observable by the LE telescope as 600. The sampling rate of LE observations will be sufficient enough to observe sudden X-ray flux increases during flares while still having the capability of monitoring the variability on time scales of years.(5) Supernovae. The LE telescope should be able to detect the theoretically predicted thermal flash lasting for $\sim 1000 \mathrm{sec}$ for the first time. Together with the optical SNe detection rate and estimates of the LE FOV we estimate the total number of SNe thermal flashes observed by the LE experiment to $\sim 10$ year. (6) AGNs. Active Galactic Nuclei will surely be one of the key targets of the LE experiment. LE will be able to monitor the behavior of the large $(\sim 1000)$ sample of AGNs providing long-term observational data with good time sampling (hours). (7) Xray transients. The LE experiment will be ideal to observe X-ray transients of various nature due to its ability to observe the whole sky several times a day for a long time with a limiting flux of about $10^{-12} \mathrm{erg} \mathrm{cm}^{-2} \mathrm{~s}^{-1}$. More and fainter X-ray transients are expected to be detected by the LE sky monitor enabling the detailed study of these phenomena. (8) Cataclysmic Variables. Cataclysmic Variables (CVs) are very active galactic objects, often showing violent long-term activity in both the optical and X-ray passband (outbursts, high/low state transitions, nova explosions) as well as rapid transitions between the states of activity. Search for the relation of the optical and X-ray activity is very important - monitoring of a large number of $\mathrm{CVs}$ is necessary to catch them in various states of activity. Most up to now X-ray observations of CVs: (i) Snapshots catching selected CVs in a particular state of activity, (ii) In most cases the transitions between the states are not covered, and (iii) Poor statistics of phenomena and objects (deeper studies available for only a few CVs). Important classes of CVs for LOBSTER are Non-magnetic dwarf novae (DNe), Supersoft X-ray sources (SSXSs), Classical novae (CNe), and Polars with soft X-ray excess.

\section{Conclusions}

Analysis and simulations of Lobster-eye X-ray telescopes have been carried out. They have 
indicated that these innovative devices will be able to monitor the X-ray sky at an unprecedented level of sensitivity, an order of magnitude better than any previous X-ray all-sky monitor. Limits as faint as $10^{-12} \mathrm{erg} \mathrm{cm}^{-2} \mathrm{~s}^{-1}$ for daily scanning observation as well as the angular resolution $<4$ arcmin in soft $\mathrm{X}$-ray range are expected to be achieved allowing monitoring of all classes of X-ray sources, not only X-ray binaries, but also fainter classes such as AGNs, coronal sources, cataclysmic variables, as well as fast $\mathrm{X}$-ray transients including gamma-ray bursts and the nearby type II supernovae.

The Lobster optics based All Sky Monitor is capable to detect around 20 GRBs and 8 XRFs yearly and this will surely significantly contribute to the related science. More details on the advantages of LE X-ray telescopes in scientific analysis of SNe are given in Sveda et al., [11]. The various prototypes of both Schmidt as well as Angel arrangements have been produced and tested successfully, demonstrating the possibility to construct these lenses by innovative but feasible technologies. Both very small Schmidt lenses $(3 \times 3 \mathrm{~mm})$ as well as large lenses $(300 \times 300 \mathrm{~mm})$ have been developed, constructed, and tested. This makes the proposals for space projects with very wide field lobster eye optics possible for the first time.

\section{Acknowledgments}

We acknowledge the support provided by the Grant Agency of the Academy of Science of the Czech Republic, grant IAAX01220701, by the Ministry of Education and Youth of the Czech Republic, projects ME918 and ME09028 and by Ministry of Industry and Trade of the Czech Republic, FD-K3/052. The investigations related to the ESA XEUS project are supported by the ESA PECS Project No. 98039. The scientific part of the study is related to the grants of the Grant Agency of the Czech Republic 102/09/0997 and 205/08/1207. We also acknowledge the collaboration with Faculty of Natural Science of the Charles University in Prague, Dr. Adam Petrušek and Petr Jan Juračka, in analyses of crayfish eyes (Figs. 1 and 2 in this paper).

\section{References}

[1] Amati, L. et al., 2004, A\&A, 426, 415.

[2] Angel J. R. P., 1979, Astroph. J., 364, 233.

[3] De Pasquale, M. et al., 2003, ApJ, 592, 1018.

[4] Fraser, G. W. et al., 2002, Proc. SPIE, 4497, 115.

[5] Frontera, F. et al., 2004, ApJ 616, 1078.

[6] Gorenstein, P., 1998, Proc. SPIE, 3444, 382.

[7] Inneman A. et al., 2000, Proc. SPIE, 4138, 94.

[8] Inneman A., et al., 1999, Proc. SPIE, 3766, 72

[9] Priedhorsky, W. C. et al.,1996, MNRAS 279, 733.

[10] Schmidt, W. K. H., 1975, NucIM, 127, 285.

[11] Sveda L. et al., 2005, in Cosmic Explosions. Springer Proceedings in Physics, Vol. 99, Eds. J. M. Marcaide and K. W. Weiler, 197. 